\title{
Generalized detection of a turbulent front generated by an oscillating grid
}

\section{Journal Article}

Author(s):

Holzner, Markus; Liberzon, Alex; Guala, Michele; Tsinober, Arkady; Kinzelbach, Wolfgang

Publication date:

2006

Permanent link:

https://doi.org/10.3929/ethz-b-000035488

Rights / license:

In Copyright - Non-Commercial Use Permitted

Originally published in:

Experiments in Fluids 41(5), https://doi.org/10.1007/s00348-006-0193-y 


\title{
Generalized detection of a turbulent front generated by an oscillating grid
}

\author{
Markus Holzner · Alexander Liberzon • \\ Michele Guala $\cdot$ Arkady Tsinober · \\ Wolfgang Kinzelbach
}

Received: 31 January 2006/Revised: 25 July 2006/ Accepted: 25 July 2006/Published online: 29 August 2006

(C) Springer-Verlag 2006

\begin{abstract}
This report presents experimental results on the propagation of a turbulent front induced by an oscillating grid starting from rest. The purpose of this preliminary investigation is to implement and validate detection methods of the turbulent/non-turbulent interface, which are based on flow measurements (velocity and vorticity) and scalar intensity, for oscillating grid turbulence. This is done using particle image velocimetry (PIV) and fluorescent dye visualization, separately. The results of both techniques describe the spreading of the turbulent front, confirming the known dependency of the front location, $H$, on time, $t$. It is demonstrated, that the level-based detection of a turbulent front can be applied to an unsteady flow, such as grid turbulence advancing into a fluid at rest.
\end{abstract}

\section{Introduction}

Many fluid flows in nature are 'partly' turbulent (Scorer 1978), which implies that fluid regions in laminar and turbulent state often co-exist close to each other. One of the basic characteristics of this type of flows is a continuous transition of fluid from laminar to turbulent state through the boundary between the two.

M. Holzner $(\bowtie) \cdot$ A. Liberzon $\cdot$ M. Guala $\cdot$ W. Kinzelbach ETH Zurich, Institute of Environmental Engineering, ETH Hönggerberg, 8093 Zurich, Switzerland e-mail: holzner@ifu.baug.ethz.ch

A. Tsinober

The Iby and Aladar Fleischman Faculty of Engineering,

Tel Aviv University, Ramat Aviv 69978, Israel
This process is commonly referred to as 'turbulent entrainment' (Tsinober 2001). For example, entrainment occurs in free shear flows (jets, plumes, wakes, mixing layers), penetrative convection in the atmosphere and in the ocean, gravity currents, avalanches and clear air turbulence.

The entrainment process was examined experimentally mainly in free shear flows (Aguirre and Catrakis 2005; Bisset et al. 2002; Catrakis et al. 2002; Corrsin and Kistler 1954; LaRue and Libby 1974; Townsend 1956; Westerweel et al. 2002 and others), and boundary layers (e.g., Chen and Blackwelder 1978; Corrsin and Kistler 1954; Kovasznay et al. 1970). In early studies, a velocity probe was placed at the edge of a turbulent flow, where its output continuously switches between a fully turbulent and a non-turbulent signal. These observations were interpreted in terms of a sharp interface separating the two regions (Corrsin and Kistler 1954). This interface is called the 'viscous sublayer' (Corrsin and Kistler 1954), as viscous diffusion of vorticity is believed to be dominant across the interface (see Tsinober 2001). At large Reynolds numbers however, the entrainment rate and the propagation velocity of the interface relative to the fluid are known to be independent of viscosity (see Townsend 1956; Tsinober 2001 and references therein). Therefore, the slow process of diffusion into the ambient fluid must be accelerated by interactions with velocity fields of eddies of all sizes, from viscous eddies to the energycontaining eddies so that the overall rate of entrainment is set by large-scale parameters of the flow (Tsinober 2001). However, it is not known how this exactly happens.

One prominent feature of the non-turbulent flow region is that it is irrotational (Corrsin and Kistler 
1954). Indeed, as confirmed in the recent studies of Bisset et al. (2002) and Westerweel et al. (2002), vorticity shows a very sharp variation at the interface. Similar to vorticity (but less steep), passive scalars also show a finite gradient across the interface (e.g., Bisset et al. 2002). For this reason, both vorticity and passive scalars were used for the identification of the outer bounds of the turbulent regions in a number of studies. For example, Kovasznay et al. (1970) used the level of one component of velocity derivatives (which is related to the level of spanwise vorticity in this case) for the separation of the vortical and non-vortical zones in the outer part of a boundary layer. Chen and Blackwelder (1978) used a heated wall to slightly increase the temperature of the turbulent flow, which could be distinguished from (cooler) non-turbulent fluid using an array of cold wires. The same approach was followed by LaRue and Libby (1974) to study the wake of a heated cylinder. Although the method of using temperature as a passive marker makes it possible to detect whether the probe is in a turbulent or non-turbulent region, it is not able to determine accurately the interface position. Westerweel et al. (2002) (see also Westerweel et al. 2005) used particle image velocimetry (PIV) and fluorescent dye simultaneously for the investigation of the turbulent/non-turbulent interface of a turbulent jet. The fluorescent dye was used in the study to detect the interface with a high spatial resolution. Recently, Aguirre and Catrakis (2005) (see also Catrakis et al. 2002) used laser-induced fluorescence for their investigation of the properties of the outer fluid interfaces of a turbulent jet. Bisset et al. (2002) used velocity fields from direct numerical simulations (DNS) of a turbulent wake behind a flat plate for the study of the turbulent/non-turbulent interface. The vorticity magnitude was employed to detect the boundaries of the turbulent regions. The authors in Bisset et al. (2002) substantiated that the advancement of the vortical interface into the irrotational flow is driven by large-scale eddy motion and stated that the zone between turbulent and non-turbulent motion is likely to be less energetic and more diffuse in the case of flows with lower mean shear across the interface. In the present experimental investigation, a turbulent/ non-turbulent interface is induced by the motion of an oscillating grid, as in Dickinson and Long $(1978,1982)$, Voropayev and Fernando (1996), among others. When a horizontal grid starts oscillating vertically in a water tank, it produces a layer of fluid in turbulent motion. This turbulent layer propagates vertically in the tank via entrainment of the irrotational fluid. Long (1978) predicted by theoretical arguments, that the depth $\mathrm{H}$ of the turbulent layer increases with time $t$ as $H \propto(k t)^{1 / 2}$, where $k$ is defined as the 'action' of the grid, a quantity with dimensions and characteristics of an eddy viscosity. Dickinson and Long (1978) confirmed this theory presenting experiments using an oscillating grid in a cylindrical tank. Later, the same authors (Dickinson and Long 1982) presented more elaborate results on experiments in a square tank with and without rotation. Voropayev and Fernando (1996) provided additional experiments in a grid-stirred cylindrical tank over a wider range of parameters and presented a semi-empirical model based on external parameters (grid frequency, oscillation amplitude, grid diameter, grid mesh size, fluid viscosity) to explain this behavior. In this and in the aforementioned studies, the flow was visualized using neutrally buoyant tracers and recorded photographically. The position of the turbulent/nonturbulent interface was determined visually from the recorded images.

For the investigation of the entrainment mechanism, three-dimensional time-resolved measurements of turbulent quantities, such as velocity and velocity derivatives at the interface, are of utmost importance. So far, vorticity was not used directly for the detection of a turbulent front in experimental studies or in transient flows, although the azimuthal vorticity component was accessed in the studies of Westerweel et al. (2002, 2005). In view of preparation for three-dimensional measurements (Particle Tracking Velocimetry), the main point of the present work is to validate the vorticity-based method for the detection of the turbulent/non-turbulent interface. Since this interface is propagating in time, the applicability of level based techniques previously employed for stationary flows, is discussed. We resolve the larger scales of the flow and concentrate on the implementation and comparison of the different techniques used for detection. This is done by using PIV to determine the two-dimensional velocity and out-of-plane vorticity components, in addition to the more commonly used flow visualization with fluorescent dye. The experimental facility and front detection techniques are described in the following Sect. 2. The results are shown in Sect. 3, followed by the discussion and conclusions in Sect. 4 .

\section{Method}

\subsection{Experimental setup}

A sketch of the oscillating grid setup is shown in Fig. 1. The grid is a fine woven screen (circular bars of $d=1$ $\mathrm{mm}$, mesh-size $d_{0}=4 \mathrm{~mm}$ ), which is installed near the upper edge of a water filled glass tank with dimensions 
of $200 \times 200 \times 300 \mathrm{~mm}^{3}$. The distance between the grid and the water surface is 50 and $100 \mathrm{~mm}$ for the different experiments and the gap between the grid and the walls is approximately $1 \mathrm{~mm}$. The grid is connected to a linear motor, which drives the vertical oscillation on a supporting frame connected to the grid through four rods of $4 \mathrm{~mm}$ in diameter. The motor, operated in a closed loop with feedback from a linear encoder, runs at a frequency of $6 \mathrm{~Hz}$ and an amplitude $\epsilon= \pm 4 \mathrm{~mm}$ for all the experiments. A time sample of the grid velocity obtained from the encoder signal is shown in the upper right corner of Fig. 1. Both the PIV and dye visualization experiments were conducted using a high-speed camera (Photron Ultima APX, $1,024 \times 512$ pixels) at a frame rate of $50 \mathrm{~Hz}$, with an exposure time of $5 \mathrm{~ms}$. The beam of a continuous 25 Watt Ar-Ion laser is expanded through two cylindrical lenses and forms a planar laser sheet less than $1 \mathrm{~mm}$ thick, which passes through the mid-plane of the tank, as it is shown schematically in Fig. 1. The field of view extended over the whole width of the tank and had the dimensions of $200 \times 100 \mathrm{~mm}^{2}$. For the PIV experiments, the camera recorded the light scattered by neutrally buoyant Polystyrene tracer particles with a diameter of $40 \mu \mathrm{m}$. This particle size is within the range of diameters typically used for such configurations [see, for example, Table 1 in Melling (1997)]. The PIV images were processed using the commercial software Insight 3.3 from TSI Inc. (TSI Inc. INSIGHT ${ }^{T M}$ 2002), with an interrogation window of $16 \times 16$ pixels, $50 \%$ overlap, yielding about 8,000 two-component velocity

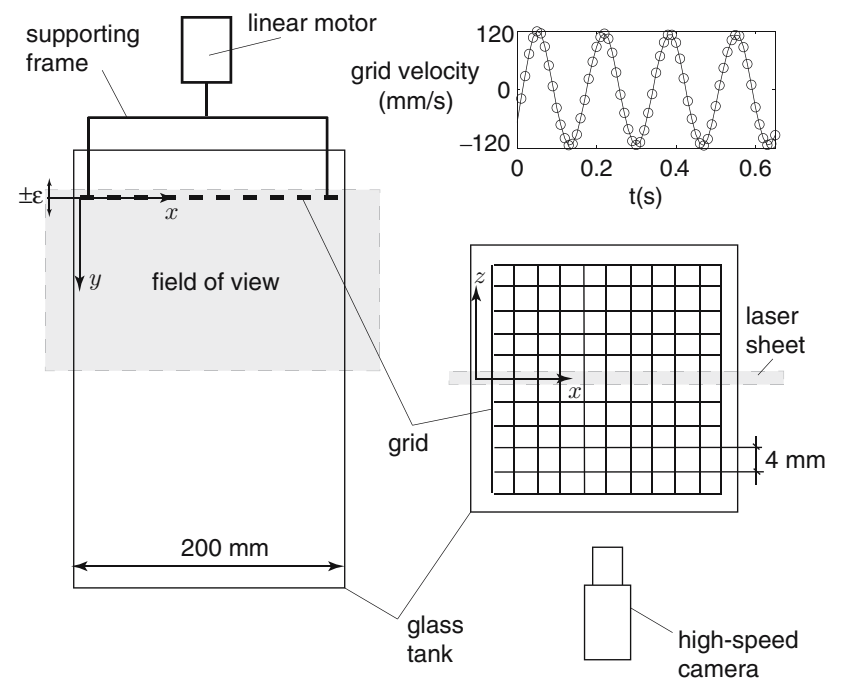

front view

top view

Fig. 1 Schematic of the experimental setup. A time sample of the grid velocity obtained from the encoder signal is shown in the upper right corner
Table 1 Values of $k$ and exponent $n$ obtained from the regression analysis for the three different detection methods

\begin{tabular}{|c|c|c|c|c|c|c|c|}
\hline \multirow[t]{2}{*}{ Exp. no. } & \multicolumn{2}{|r|}{ Symbol } & \multirow{2}{*}{$\frac{k\left(\mathrm{~cm}^{2} / \mathrm{s}\right)}{\text { Dye }}$} & \multirow[t]{2}{*}{$n$} & \multirow[t]{2}{*}{$R^{2}$} & \multirow[t]{2}{*}{$k\left(\mathrm{~cm}^{2} / \mathrm{s}\right)$} & \multirow[t]{2}{*}{$n$} \\
\hline & & & & & & & \\
\hline $1^{*}$ & & $\circ$ & 1.2 & 0.43 & 0.87 & & \\
\hline $2 *$ & & $x$ & 0.73 & 0.56 & 0.95 & & \\
\hline 3 & & $\nabla$ & 0.80 & 0.63 & 0.92 & & \\
\hline 4 & & $\bullet$ & 1.4 & 0.42 & 0.88 & & \\
\hline \multirow[t]{2}{*}{$\langle\cdot\rangle$} & & - & 0.96 & 0.53 & 0.99 & & \\
\hline & & \multicolumn{2}{|c|}{ Velocity } & \multicolumn{4}{|c|}{ Vorticity } \\
\hline 5 & $\circ$ & 1.0 & 0.51 & 0.91 & 0.82 & 0.53 & 0.92 \\
\hline 6 & $x$ & 0.73 & 0.52 & 0.89 & 1.0 & 0.52 & 0.93 \\
\hline 7 & $\nabla$ & 0.52 & 0.65 & 0.94 & 0.58 & 0.66 & 0.95 \\
\hline 8 & $\bullet$ & 0.97 & 0.51 & 0.96 & 0.87 & 0.55 & 0.95 \\
\hline $9 *$ & + & 0.82 & 0.49 & 0.92 & 0.99 & 0.45 & 0.89 \\
\hline $10^{*}$ & $*$ & 0.83 & 0.49 & 0.95 & 0.63 & 0.56 & 0.91 \\
\hline $11^{*}$ & $\diamond$ & 1.0 & 0.46 & 0.93 & 1.0 & 0.46 & 0.90 \\
\hline$\langle\cdot\rangle$ & - & 0.76 & 0.54 & 0.98 & 0.78 & 0.54 & 0.98 \\
\hline
\end{tabular}

$R^{2}$ is the correlation coefficient. The values in the rows of the table marked with $\langle\cdot\rangle$ refer to the regression of the averaged curve over experiments 1-4 and 5-11, respectively. For experiments marked by an asterisk, the distance between the free surface and the grid was $10 \mathrm{~cm}$, for all other experiments it was $5 \mathrm{~cm}$

vectors per realization, denoted as $u$ and $v$ in $x$ and $y$ direction (Fig. 1), respectively. During the post-processing the standard global outlier and local median filters were applied and about $5 \%$ of erroneous vectors were found, removed and linearly interpolated by using the values of the nearest neighbors. The $\omega_{z}$ (outof-plane) vorticity component was calculated using a least squares differentiation scheme (e.g., Raffel et al. 1998). For the flow visualization experiments, the fluid in proximity of the grid was marked with fluorescent dye before oscillation started. We used an array of syringes and did several tests injecting the dye in the whole region above the grid or just into a small region and observed that the details of injection did not influence the final outcome. The fluorescent dye (Uranin) has a Schmidt number of approximately 2,000, so that the molecular diffusion of the dye is much smaller than its turbulent mixing. The recording time for the experiments was set to $30 \mathrm{~s}$, well above the average travel time of the turbulent front through the field of view $(\sim 20 \mathrm{~s})$. The region of grid oscillation, i.e., $y=$ $\pm \epsilon$ (see Fig. 1) was excluded from the PIV analysis. In total, four experiments were carried out for the case of dye visualization and seven for PIV measurements, respectively. We did two sets of experiments to investigate the influence of the free surface on the front propagation (see also Voropayev and Fernando 1996 for a theoretical estimate of this influence). In the first set, the distance between the grid and the water surface 
is $5 \mathrm{~cm}$, in the second set the distance is $10 \mathrm{~cm}$ (experiments marked by an asterisk in Table 1).

\subsection{Detection of the turbulent/non-turbulent interface}

\subsubsection{Detection through scalar}

The instantaneous concentration field of the fluorescent dye is commonly used for the detection of the interface (e.g., Westerweel et al. 2002). We used the procedure described in Westerweel et al. (2002) to detect the interface from the images of the concentration field (a typical image is shown in Fig. 2a). The four main steps of the procedure are illustrated in Fig. 2 and summarized as follows:

1. A median filter is applied to the image in order to remove noise (single-pixel objects) in the background.

2. The gray level intensity images are transformed into binary images by means of a threshold, i.e., all pixels with a gray value above the threshold are labelled with ' 1 ', the remaining pixels are labelled with ' 0 ' (Fig. 2b). A fixed gray-value threshold (normalized by the maximum value of 255), $c_{I}$, is chosen using the technique proposed by the authors in Prasad and Sreenivasan (1989): in each image, the mean gray level intensity of all the pixels above the varying threshold is calculated. This conditionally sampled mean gray level intensity is shown of Fig. 3a, where the different curves correspond to different times (in the figure, the axes are normalized by the maximum gray-value).
As the initially highly concentrated dye diffuses, the mean gray level is decreasing in time. We also computed the time averaged curve and used one fixed threshold for all images. In the time averaged curve shown in Fig. 3b, we detect two nearly linear regions indicated by two dashed lines. According to Prasad and Sreenivasan (1989), the intersection point of the two dashed lines determines the value of the threshold (in our case $c_{I}$ ranged from 0.23 to 0.26 for the different experiments).

3 . In the binary images we observed patches of entrained ambient fluid in the turbulent region (which appear as 'holes') and 'islands' of marked fluid outside of the continuous region. The detached patches of dye (see arrow in Fig. 2b) were removed (Fig. 2c).

4. The boundary of the continuous object is detected as the lowest point $\max (y)$ at each $x$ (see Fig. 2d). The resulting discontinuous line is only a rough representation of the presumably continuous and smooth interface, reflecting mainly the large scales. However, for our purpose of analyzing the propagation of the turbulent front, this level of representation is regarded as sufficiently accurate.

Given the large Schmidt number of the fluorescent dye and the limited resolution of the camera, the smallest scales of the dye interface are not resolved by our measurements, as discussed in the following. Usually the scales of the flow quantities and the concentration field are defined on the basis of dimensionless parameters, such as the Reynolds number of the flow. It is difficult to define a Reynolds number associated with our experiments, since the flow is transient and the scales of the flow are expected to
Fig. 2 A typical example of raw and processed images of fluorescent dye. a Raw, gray level image, b binary image, c single object, d object boundary
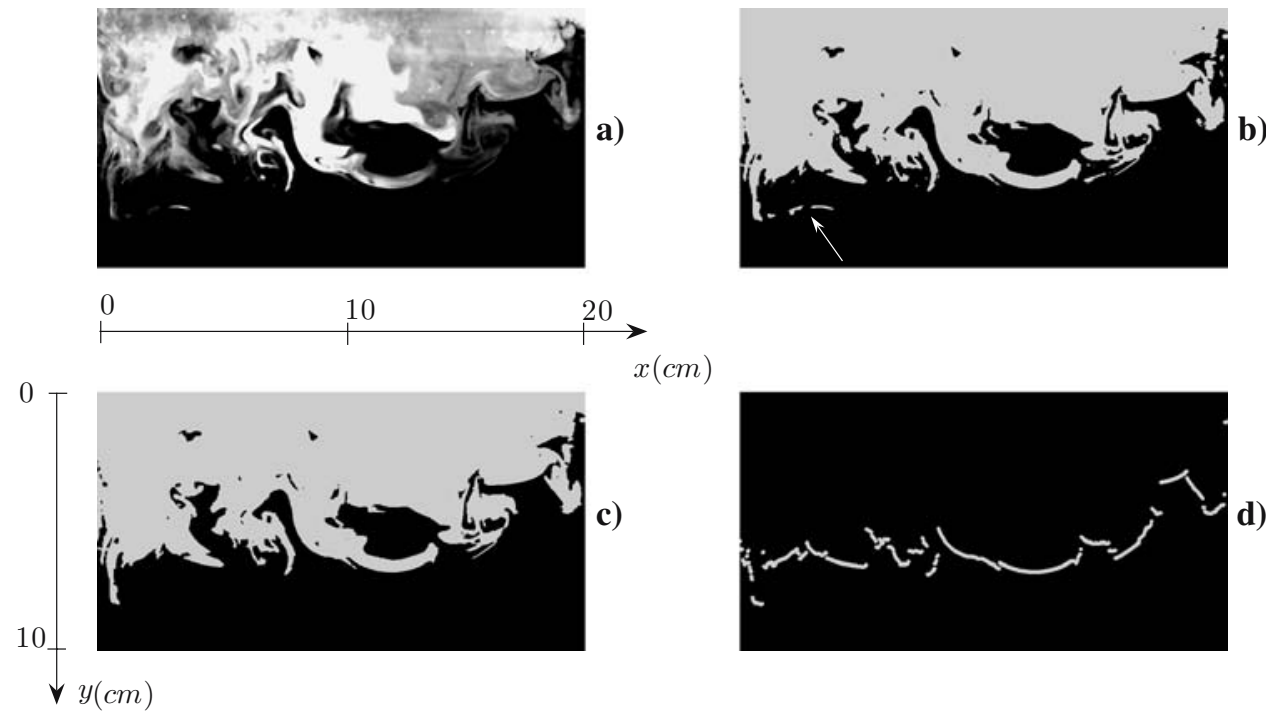

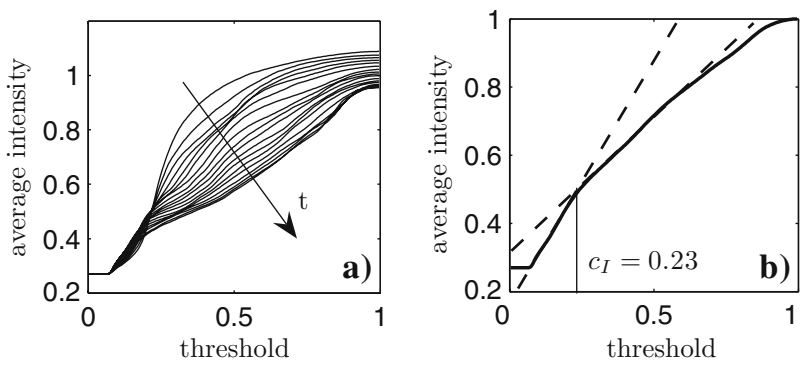

Fig. 3 Average intensity of all pixels above the threshold as a function of the threshold (see Prasad and Sreenivasan 1989) for a given experiment: a variation in time $(0-20 \mathrm{~s})$; b time averaged curve

change in space and time. Voropayev and Fernando (1996) provided a Reynolds number based on the geometry and forcing parameters of the grid for their experiments. According to their definition (Eq. 9 in Voropayev and Fernando 1996), the global Reynolds number of the flow is $R e=1,800$ for our experiments. The smallest scales of the dye concentration field are of the order of the Batchelor scale, defined as

$\eta_{B}=\frac{\eta}{\sqrt{S c}}$

where $\eta$ is the Kolmogorov scale and $S c$ is the Schmidt number. The Kolmogorov scale is expected to be smallest in the vicinity of the grid, where turbulence intensities are high. The Kolmogorov scale can be calculated using

$\eta=\frac{v^{3 / 4}}{\left\langle\epsilon^{1 / 4}\right\rangle}$

where $v$ is the viscosity of the fluid and $\langle\epsilon\rangle$ is the mean dissipation rate. Here, by the term 'mean' we refer to the average of a quantity over a period that is longer than the turbulence time scales in a given location of the flow domain. As mentioned previously, the flow properties change in space and time. However, once the turbulent front is far from the grid, the turbulent quantities close to the grid are expected to be statistically stationary. This was verified by our measurements. We selected a thin horizontal window $1 \mathrm{~cm}$ away from the grid and averaged the velocity gradients from the PIV measurement over the last few seconds of the experiment. With the $2 \mathrm{D}$ approximation, $\langle\epsilon\rangle$ was estimated to be of the order of $\mathcal{O}\left(10^{-4}\right) \mathrm{m}^{2} / \mathrm{s}^{3}$, which leads to an estimate of $\eta$ of about $0.3 \mathrm{~mm}$ in that region. Hence, the Batchelor scale can be estimated to be of the order of $\mathcal{O}\left(10^{-2}\right) \mathrm{mm}$. The large scales of the dye concentration field will be comparable to the large scales of the flow, which grows from a size that is comparable to the grid mesh size in the vicinity of the grid to several cm's further away. A good estimate for the upper limit of the large scale of the concentration field in the considered flow region could be the half width of the tank, i.e., $10 \mathrm{~cm}$. Given that the resolution for the dye visualization is about $0.2 \mathrm{~mm} /$ pixel for our experiments, the smallest scalar scales are expected to be one order of magnitude smaller than the smallest resolved scales.

\subsubsection{Detection through velocity and vorticity}

It is known (e.g., Corrsin and Kistler 1954) that a sharp interface separates irrotational, non-turbulent fluid from vortical turbulent fluid. That is why vorticity is an adequate quantity for the detection of the turbulent/ non-turbulent interface (e.g., Bisset et al. 2002). Since also velocity fluctuations, among other turbulent quantities, decrease sharply at the interface (although less steeply than vorticity, e.g., Bisset et al. 2002), the velocity magnitude was also tested for the detection. Differently from vorticity, velocity fluctuations in the non-turbulent region are often non-zero, because of irrotational fluctuations induced by large-scale turbulent motions [previously observed in Bisset et al. (2002) and references therein]. Nevertheless, these fluctuations are typically smaller than the ones inside the turbulent region and they decay very rapidly with distance from the interface. The detection was based on the instantaneous two-dimensional velocity or vorticity component realizations and implemented as a level-based method. A typical vector plot of velocity, overlayed by the contours of the instantaneous vorticity component $\omega_{z}$ is shown in Fig. 4.

As in all level-based methods, the threshold has to be set appropriately, but there is no unique, 'best' choice. For instance, the 'zero-threshold' is not applicable, because noise associated with PIV measurements and analysis leads to non-zero vorticity and velocity in the irrotational region. Nevertheless, due to the steep spatial gradient of turbulent quantities across the interface (see e.g., Bisset et al. 2002; Westerweel et al. 2002), genuine vorticity/velocity could be distinguished from the noise. We estimated the noise level of vorticity from the root-mean-square (r.m.s.) values of $\omega_{z}$ in the lower (non-turbulent) part of the field of view, for the first few seconds of the experiment, when the flow was still undisturbed. It was found that the noise level is of the order of $\mathcal{O}(0.1) s^{-1}$. Similarly, the noise level of velocity was estimated to be of the order of $\mathcal{O}(0.5) \mathrm{mm} / \mathrm{s}$. The boundary of the turbulent region is detected similarly to step 4 in the previous section. For each time instance $t$, and for each $x$ the turbulent 


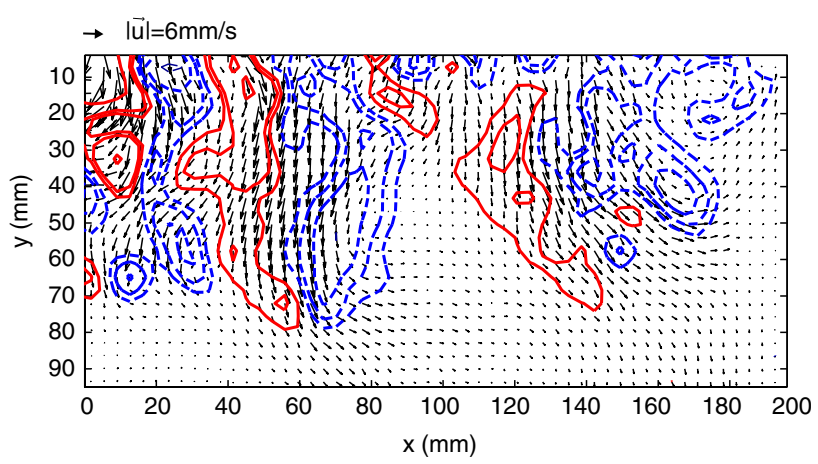

Fig. 4 An example of an instantaneous PIV realization. Vectors show the direction and the magnitude of the velocity field and contours are of $\omega_{z}$. Solid lines denote the positive values and the dashed lines are for the negative values (the lowest level is $0.3 \mathrm{~s}^{-1}$ )

boundary is the lowest point, $y^{*}(x, t)$, in which the magnitude of the signal exceeds a fixed (for all times and $x$ locations) threshold ( $c_{\omega}$ and $c_{u}$ for vorticity and velocity, respectively). The procedure is depicted in Fig. 5, in which three typical vertical profiles of vorticity are shown, along with the threshold and the detected points $y^{*}(x, t)$.

Contours of $\omega_{z}$ were plotted for many time steps (as for example shown in Fig. 4) and it was found that a level of the threshold of four times the noise level (for both vorticity and velocity) is the best to delineate the vortical regions for all experiments. For thresholds above this level, some low vorticity regions were left out, while below this level the profile shows excursions into non-rotational regions. Moreover, we applied the test proposed by Bisset et al. (2002) in which the threshold $c_{\omega}$ was evaluated by analyzing the conditionally averaged (over time and horizonal coordinate $x)$ vertical profiles of $\left|\omega_{z}(\hat{y})\right|$, where $\hat{y}=y-y^{*}$. The

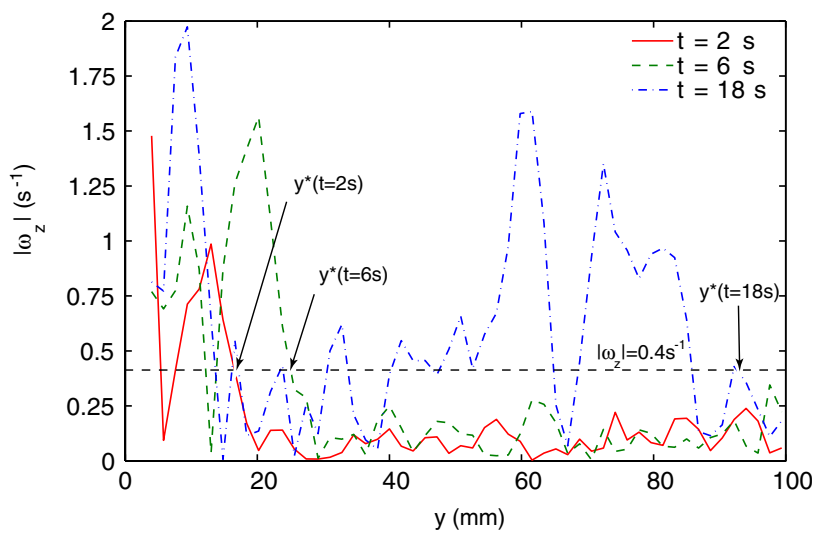

Fig. 5 Vertical profiles of vorticity magnitude for three different time instances at $x=50 \mathrm{~mm}$ conditionally averaged vertical profiles of $\left|\omega_{z}(\hat{y})\right|$ and $|\vec{u}(\hat{y})|=\sqrt{u(\hat{y})^{2}+v(\hat{y})^{2}}(|\cdot|$ denotes the absolute value $)$ are shown in Fig. 6 . Since the vertical velocity component is of interest because of its relation to the entrainment rate (e.g., Westerweel et al. 2005), also $|v(\hat{y})|$ is plotted in the figure. Note that the steepness of the gradient of the profile at the interface (the location where vorticity/velocity magnitude changes from the low to the high level) is the important parameter of this test, commonly related to the sharpness of the identified interface (e.g., Bisset et al. 2002; Corrsin and Kistler 1954). As expected, the steepness of the gradient of the velocity profiles is less pronounced than the one of vorticity.

Figure 7 shows the spatial distributions of the magnitude of vorticity and velocity, respectively (high gray level corresponds to high intensity). The detected interface is visualized on both panels by assigning white markers to the position of $y^{*}(x, t)$ for three different time instances. We observe that the propagation of the turbulent front, as detected by vorticity and velocity, is very similar in both right and left panels. The slight differences can be associated with the fact that the PIV measurements yield two velocity components and only one vorticity component, i.e., the outof-plane component. The measured component of vorticity can be zero in regions where the other two components are not. This leads to holes in the turbulent regions visible in the vorticity magnitude plots of Fig. 7. This effect is less pronounced in the case of velocity. It is also visible in the figure, that the contour obtained by using the velocity is more continuous than the one for vorticity. As we shall show below, the lack of 'continuity' is not problematic for the present analysis as we concentrate on the overall front propagation in terms of a horizontal average.

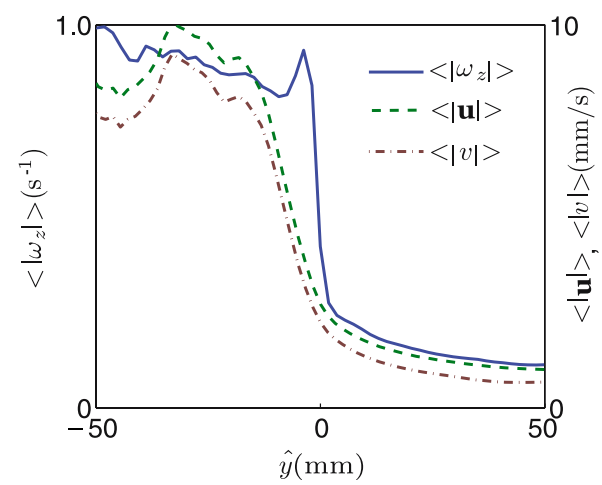

Fig. 6 Conditionally sampled vertical profiles of the magnitudes of vorticity (left axis, solid line) and of velocity vector and vertical velocity component (right axis, dashed and dashed dotted lines) 
Fig. 7 Vorticity (left panel) and velocity (right panel) magnitude maps for three time instances, a $t=2 \mathrm{~s}$, b $t=6 \mathrm{~s}, \mathbf{c} t=18 \mathrm{~s}$. The white spots represent the detected front, i.e., $y^{*}(x, t)$
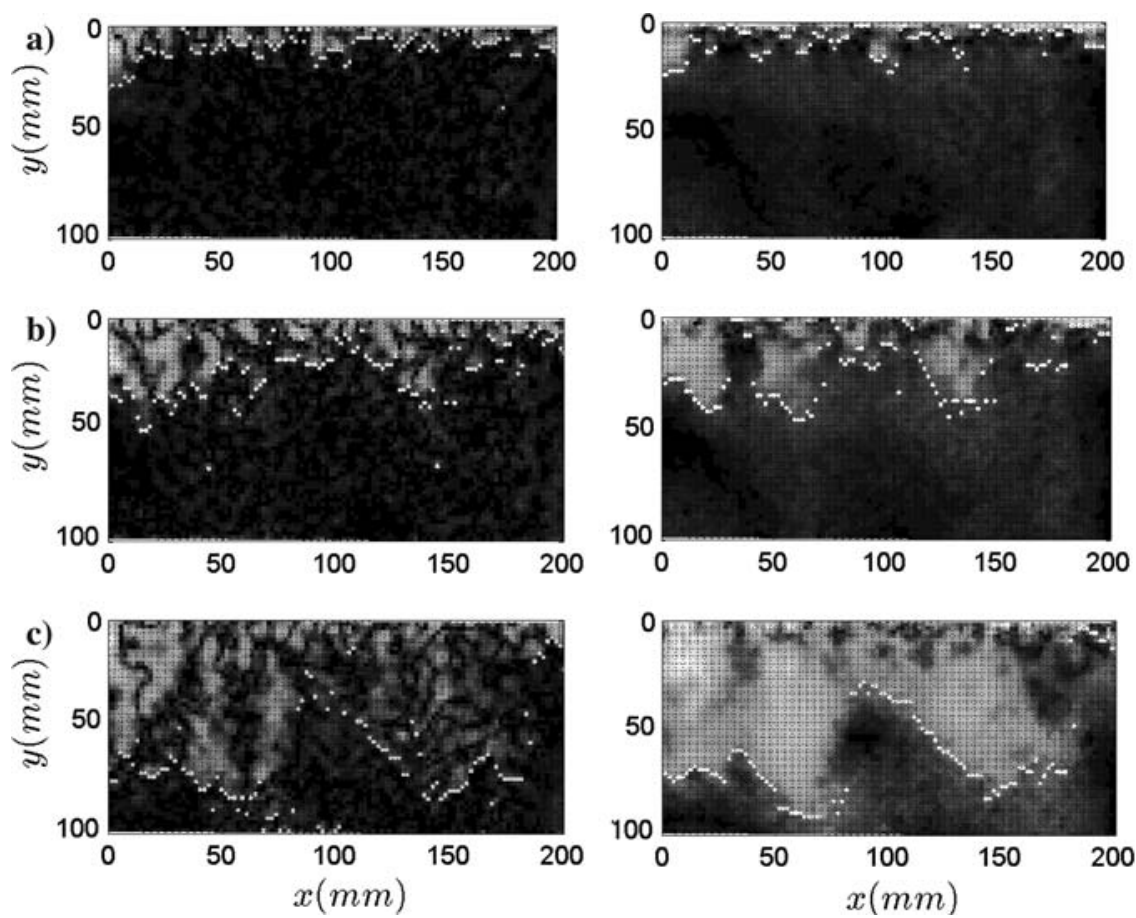

\section{Results}

The present study focuses on the propagation (in time) of the turbulent/non-turbulent interface, detected using the techniques described above. When the grid starts to oscillate each grid bar generates, alternatively, small regions of positive and negative vorticity, because of the no-slip condition at the surface of the grid elements (Voropayev and Fernando 1996). The induced velocity field advects the vorticity away from the boundary. It was inferred by Voropayev and Fernando (1996) (among others) that in the initial stage of the grid movement, when the typical width of the small jets/vortices is less than the grid mesh size $d_{0}$, there is little interaction between them. However, as these structures grow with time (and their size becomes comparable to $d_{0}$ ) these jets/vortices start to interact and the vortical flow becomes turbulent. In the semiempirical model of Voropayev and Fernando (1996), the expression for the initial stage, $t_{0}$, is given as

$t_{0}=\frac{d_{0}^{2}}{2 c_{1}^{2} c_{2} R e^{3 / 5} v}$,

where $v$ is the kinematic viscosity of water, $\mathrm{c}_{1} \simeq 2.5$ and $c_{2} \simeq 0.14$ are two constants associated with the shape of the grid bars (see (Voropayev and Fernando 1996). For our setup, Eq. 3 yields $t_{0} \simeq 0.1 \mathrm{~s}$. For the later times it was predicted by Long (1978) and verified in many subsequent studies (e.g., Dickinson and Long 1978; Voropayev and Fernando 1996) that the interface propagates as $H \propto(k t)^{1 / 2}$, where $H$ is the distance from the grid to the interface (typically $H$ and $t$ are given in dimensional units of centimeters and seconds, respectively).

The propagation of the turbulent front, as it is detected by the aforementioned methods, is depicted in Fig. 8a-c. Here each point of $H(t)$ is the average of $y^{*}(x, t)$ over $x$ for the different experiments. In addition to the values for each experiment marked by symbols, the trend line, which is an average of $H(t)$ for the different experiments, is plotted in the figure as a continuous line. It can be noticed that the single experiments show significant scatter due to the direct influence of the grid oscillation. However, this scatter is not larger than the range of variation between the different experiments. Some experiments show a somewhat smaller slope within the first $1-2 \mathrm{~s}$ of the experiment, visible also in the trend lines. We understand that the theoretical prediction of $0.1 \mathrm{~s}$ for the initial stage, where turbulence is not yet developed, may underestimate the real time span needed. The slopes of the plotted curves were estimated by regression analysis (similar to Dickinson and Long 1978), using:

$H=k^{\frac{1}{2}} t^{n}$

or

$\ln (H)=n \cdot \ln (t)+\frac{1}{2} \ln (k)$. 

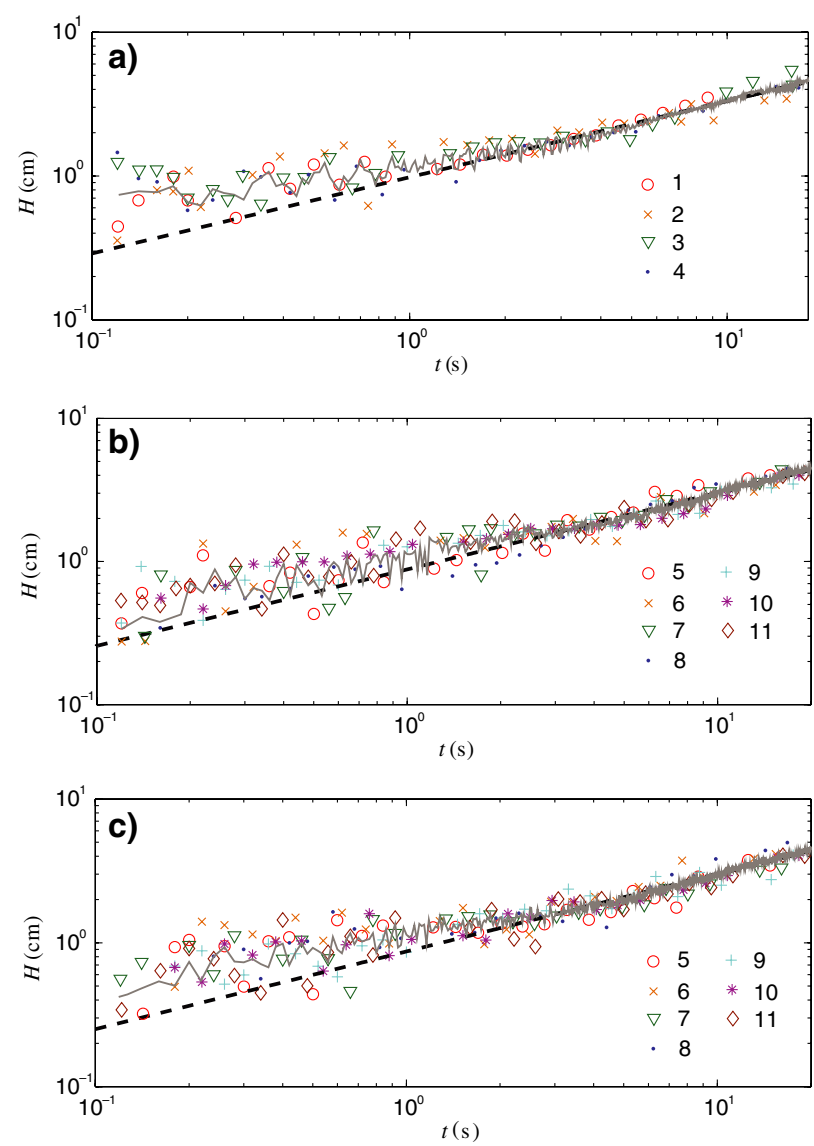

Fig. 8 Vertical position of the interface, $H$, versus time, detected by using a dye concentration, b velocity and c vorticity. The different experiments are listed in Table 3. The continuous and dashed lines represent the average curve over the experiments and its best fit estimated by regression analysis (Eq. 4), respectively. For the sake of clarity the number of plotted data points is reduced

The results of the regression analysis are summarized in Table 1 for the dye visualization and PIV experiments, respectively. The table also includes the calculated values for the trend lines. Their best fits are plotted as dashed lines in Fig. 8a-c. We observe that the theoretical value for the slope of 0.5 is reasonably met by the experiments with the two extreme values of 0.42 and 0.66 in experiments 4 and 7 , respectively. Also, we observe that the slopes of the averaged curves are slightly higher than the theoretical value. This is probably due to the limited number of experiments. The slopes found by the detection through velocity and vorticity are not equal, due to the different characteristics of the detected interface mentioned above.

As mentioned in Sect. 2, we carried out two sets of experiments to test the influence of the distance between the water surface and the grid on the front propagation. In the first set the distance between the grid and the water surface was $10 \mathrm{~cm}$ (experiments labelled by an asterisk in Table 1) and in the second set it was $5 \mathrm{~cm}$, respectively. It is visible in Table 1 that there is no systematic dependence of the values on the distance between the grid and the water surface. It is also noteworthy that the theory of Long (1978) is valid for a semi-infinite space, in which the effect of the bottom or the side walls was not taken into account. In our experiments, strong vorticity was observed shedding from the grid border and propagating along the walls. This is also visible in the vorticity contours of Fig. 4. Similar observations can be found in Voropayev and Fernando (1996). We limited the influence of this effect excluding $2 \mathrm{~cm}$ of horizontal distance on either side of the observation window from the computation of $H(t)$. By using this exclusion, generally the estimated slopes were closer to the $H \propto(k t)^{1 / 2}$ law, than the ones obtained over the whole width of the container. The mean value of the slopes obtained by all three detection methods is $\langle n\rangle=0.52$ with a standard deviation of 0.07 (or $14 \%$ ). In the above discussion, the regression analysis was done using $k$ as a free parameter. However, $k$ can be characterized in terms of grid geometry and forcing parameters (Dickinson and Long 1978, 1982) and should not vary between the experiments. If we fix $k$ to the mean value over the coefficients obtained from the vorticity based detection, i.e., $\langle k\rangle=0.84 \mathrm{~cm}^{2} / \mathrm{s}$, the mean value of the slope is almost the same, $\langle n\rangle=0.53$, but the standard deviation is reduced to 0.04 (or $8 \%$ ).

The important observations are: (1) the results for the three different quantities are consistent with each other and with the existing literature; (2) applying the threshold evaluation technique described above, two-dimensional velocity data and the derived onecomponent vorticity fields are valid for a reliable and robust detection of the propagating turbulent front.

\section{Conclusions}

The propagation of the turbulent/non-turbulent interface, generated by an oscillating grid starting from rest, was analyzed using flow visualization and PIV. Both techniques have already been applied for the detection of the turbulent front for flows with significant mean shear. The present study shows that they are also valuable tools for oscillating grid experiments. Some important properties of the front were confirmed, such as the steep gradient of vorticity at the interface and the propagation velocity of the front. The theoretically predicted $(k t)^{1 / 2}$ law of propagation of the turbulent front in time could be verified with both experimental techniques. 
The similarity of the results derived from concentration, velocity and vorticity measurements and the consistency with available data, suggests that it is possible to utilize the level-based interface detection method in the future three-dimensional Lagrangian measurements of the interface. The robust determination of the threshold will allow for detection of the Lagrangian trajectories that cross the sharp interface between turbulent and non-turbulent regions.

Acknowledgment This work was supported by ETH Grant No. 0-20151-03.

\section{References}

Aguirre RC, Catrakis HJ (2005) On intermittency and the physical thickness of turbulent fluid interfaces. J Fluid Mech 540:39-48

Bisset DK, Hunt JCR, Rogers MM (2002) The turbulent/nonturbulent interface. J Fluid Mech 451:383-410

Catrakis JC, Aguirre RC, Ruiz-Plan Carte J, Thayne RD, McDonald BA, Hearn JW (2002) Large-scale dynamics in turbulent mixing and the three-dimensional space-time behaviour of outer fluid interfaces. J Fluid Mech 471:381408

Chen CHP, Blackwelder RF (1978) Large-scale motion in a turbulent boundary layer: a study using temperature contamination. J Fluid Mech 89:1-31

Corrsin S, Kistler AL $(1954,1955)$ The free-stream boundaries of turbulent flows. NACA, TN-3133, TR-1244, pp 1033-1064
Dickinson SC, Long RR (1978) Laboratory study of the growth of a turbulent layer of fluid. Phys Fluids 21(10):1698-1701

Dickinson SC, Long RR (1982) Oscillating-grid turbulence including the effects if rotation. J Fluid Mech 126:315-333

Kovasznay LSG, Kibens V, Blackwelder RF (1970) Large-scale motion in the intermittent region of a turbulent boundary layer. J Fluid Mech 41:283-325

LaRue JC, Libby PA (1974) Temperature fluctuations in the plane turbulent wake. Phys Fluids 17:1956-1967

Long RR (1978) Theory of turbulence in a homogeneous fluid induced by an oscillating grid. Phys Fluids 21(10):1887-1888

Melling M (1997) Tracer particles and seeding for particle image velocimetry. Meas Sci Technol 8:1406-1416

Prasad RR, Sreenivasan KR (1989) Scalar interfaces in digital images of turbulent flows. Exp Fluids 7:259-264

Raffel M, Willert C, Kompenhans J (1998) Particle image velocimetry-a practical guide. Springer, Berlin Heidelberg New York, Germany

Scorer RS (1978) Environmental aerodynamics. Halsted Press, New York

Townsend AA (1956) The structure of turbulent shear flow. Cambridge University Press, London

TSI Inc. INSIGHT ${ }^{T M}$ 3.3 PIV Evaluation Software, 2002, TSI Inc., Shoreview (MN), USA

Tsinober A (2001) An informal introduction to turbulence. Kluwer, The Netherlands

Voropayev SI, Fernando HJS (1996) Propagation of grid turbulence in homogeneous fluids. Phys Fluids 8(9):2435-2440

Westerweel J, Hoffmann T, Fukushima C, Hunt JCR (2002) The turbulent/non-turbulent interface at the outer boundary of a self-similar turbulent jet. Exp Fluids 33:873-878

Westerweel J, Fukushima C,Pedersen JM, Hunt J (2005) Mechanics of the turbulent-nonturbulent interface of a jet. Phys Rev Lett 95:174501 